\title{
E-GOBIERNO Y CAMBIO INSTITUCIONAL: EL IMPULSO DE LA TRANSVERSALIDAD EN LA ADMINISTRACIÓN PÚBLICA*
}

\author{
Miquel Salvador \\ Universitat Pompeu Fabra, España \\ miquel.salvador@upf.edu
}

\section{RESUMEN}

El artículo plantea la conexión entre el impacto organizativo del gobierno electrónico y el concepto de transversalidad, enfatizando en su definición los componentes de integración, coordinación y comunicación interdepartamental, así como la idea de proponer unos objetivos compartidos que no son propios de ningún ámbito de la organización sino generales del conjunto. A continuación se discuten, en clave neoinstitucionalista, su concreción en el campo de la organización, los roles directivos y los estilos de relación. La cultura de la transversalidad se plantea como un referente para el impulso de estrategias de gobierno electrónico en las administraciones públicas.

Palabras clave: Gobierno electrónico, Transversalidad, Cambio organizacional, Cultura organizativa.

* Una versión preliminar de este artículo fue presentada y discutida en el IX Congreso Español de Ciencia Política y de la Administración organizado por la Asociación de Ciencia Política y de la Administración, celebrado en Málaga los días 23 a 25 de Septiembre de 2009, en el "Grupo de trabajo 5.2: Innovación en la gestión pública: e-Gobierno y cambio institucional a través de la tecnología”. El autor agradece a los comentaristas y a los participantes en el mismo sus valiosas aportaciones para la mejora del documento final. 


\title{
E-GOVERNMENT AND INSTITUTIONAL CHANGE: THE PROMOTION OF TRANSVERSALITY IN PUBLIC ADMINISTRATION
}

\begin{abstract}
This article illustrates the connection between the organizational impact of e-Government and the concept of transversality, emphasizing in its definition the components of integration, coordination and interdepartmental communication, as well as the idea to suggest shared goals which are not specific to any area, but common to the organization as a whole. Their implementation in the field of organization, management roles and types of relationships are discussed below in a neo-institutionalist approach. The culture of transversality is posed as a reference for the promotion of e-Government strategies in public administrations.
\end{abstract}

Keywords: E-Government, Transversality, Organizational change, Organizational culture. 


\section{INTRODUCCIÓN}

La incorporación de las tecnologías de la información y la comunicación (TIC) en la diversidad de subsistemas de la gestión pública, desde el diseño de instrumentos de seguimiento y evaluación a la gestión de los recursos humanos, ha convertido a los instrumentos asociados a este ámbito en elementos imprescindibles en toda organización pública. Con diversidad de apuestas organizativas para su concreción y complicadas concreciones operativas que suelen distar de los objetivos y expectativas generados, las TIC forman parte ya de los ámbitos de la denominada tecnoestructura de las administraciones públicas.

Sin embargo, su evolución no ha sido lineal sino que parece asimilarse a un ciclo que se ha movido desde una visión de cambio interno a una visión que primaba la relación hacia el exterior, para retornar de nuevo a revalorizar la necesidad de generar cambios internos, aunque en una nueva fase caracterizada por una visión estratégica e integral.

Avanzada la segunda mitad del siglo XX, la incorporación de las TIC a la gestión pública se planteó con una orientación interna, básicamente asociada a la mejora de la eficiencia y la productividad del sector público, en un estadio que algunos autores han denominado informatización (Bellamy y Taylor I 998). Sin un alcance integral ni un enfoque estratégico, a este estadio se asocian la introducción de aplicativos sectoriales orientados a mejorar procesos concretos de la actividad administrativa, con una notable impronta de enfoques propios de la ingeniería industrial aplicada, con menor o mayor éxito, a una realidad administrativa en constante cambio (más allá del tecnológico).

En paralelo, aunque sin abandonar los objetivos de mejora interna planteados en la fase inicial de orientación interna, muchos gobiernos apostaron por el potencial de relación externa que ofrecían las TIC, especialmente a partir de la extensión de Internet y del surgimiento de nuevos usos sociales de relación y comunicación (Dunleavy y Margetts 2007). En este segundo enfoque de carácter más externo, se destaca la orientación al ciudadano de las administraciones públicas y con ella la necesidad de ofrecer contenidos de información y servicios públicos electrónicos adecuados a los diferentes colectivos. Aunque algunos autores denominan a esta nueva orientación como administración electrónica, realmente su concreción en muchas administraciones públicas distó de incluir realmente planteamientos de cambio integral o conexiones realmente consistentes con la orientación anterior, por lo que proponemos denominarla orientación externa. El surgimiento de las primeras páginas web de las administraciones públicas a mediados de los años noventa del 
siglo XX constituye un indicador del nuevo enfoque (Criado y Ramilo 200 I, Salvador, Cortés y Sánchez 2004. Mesa del Olmo 2007).

El rasgo relevante de esta evolución, tal como se ha apuntado, es la falta de coincidencia de los avances realizados en la fase inicial interna (todavía incipientes) con las apuestas por mejorar la dimensión externa de la segunda (mucho más reciente pero con ritmos de extensión mucho más acelerados), denotándose la ausencia de planteamientos integrales que permitiesen generar sinergias y, en definitiva, hacer sostenibles las estrategias de mejora del servicio público planteadas a través de la incorporación de las TIC.

A las orientaciones anteriores se suma, especialmente a partir de los primeros ańos del siglo XXI, un nuevo enfoque de carácter más relacional con el que, destacando el concepto de la interoperabilidad, se propone utilizar las TIC como instrumento que debería facilitar la relación entre administraciones públicas (Comisión Europea 2006) y entre éstas y otras organizaciones implicadas en la prestación de servicios públicos, en la denominada dimensión relacional.

Más allá de las importantes dificultades tecnológicas asociadas al desarrollo de cada uno de los tres enfoques apuntados (interno, externo y relacional), se planeta la conveniencia de desarrollar estrategias integradas que faciliten la consolidación de los avances logrados en los diferentes ámbitos o, en otros términos, estrategias de gobierno electrónico (Salvador 2005a, 2005b).

Pero cuestión aparte es el encaje de estos impulsos transformadores basados en los diferentes enfoques de uso de las TIC con las dinámicas internas y relacionales de las administraciones públicas. Unas administraciones públicas cuya lógica de actuación no propiciaba los enfoques integrales y transversales que se podrían asociar a la introducción de las TIC para la mejora del valor público que éstas deberían ofrecer a la sociedad.

Estas dificultades de ajuste pueden interpretarse en términos de convivencia de instituciones diversas en el seno de unas administraciones públicas diseñadas para responder a un tipo de entornos y retos distintos a los actuales. El diseño dominante ha tendido a la multiplicación de organizaciones sectoriales canalizadas en estructuras de carácter jerárquico, fruto tanto de lógicas políticas como lógicas administrativas orientadas a la creación de unidades profesionales y operativas que se articulaban mediante planteamientos basados en la sectorialización y la jerarquía.

Estos planteamientos condicionan en buena medida la cultura organizativa dominante a las administraciones públicas. Como resultado 
de este proceso se han creado estructuras administrativas con unidades muy especializadas que generan procesos de decisión y de gestión fuertemente fragmentados (Ramió, Salvador y García 2007). Aunque se trata de un diseño que eventualmente puede ser eficiente, resulta cada vez menos eficaz puesto que las nuevas políticas y servicios fruto de los cambios del entorno y de las propias dinámicas internas de funcionamiento de las administraciones públicas requieren de soluciones transversales.

Pese a que el concepto haya podido considerarse en determinados contextos como una moda, el requerimiento de dar respuestas coherentes e integradas a los nuevos retos que se plantean en la multiplicidad de campos de actuación de las administraciones públicas ha convertido al concepto de transversalidad en una necesidad y una oportunidad para transformar la gestión pública.

En este contexto, más allá de la necesaria clarificación del concepto, el artículo se plantea exponer una serie de reflexiones sobre las opciones para desarrollar estrategias que, de forma realista y pragmática, den contenido y concreción al concepto de transversalidad y a sus vinculaciones potenciales con el gobierno electrónico.

\section{SOBRE EL CONCEPTO DE TRANSVERSALIDAD}

El concepto transversalidad ha hecho fortuna en el léxico de los gestores públicos y existe un amplio consenso al considerarlo como un cambio necesario para implementar políticas públicas más eficaces que superen las limitaciones de unas estructuras demasiado sectorializadas. Pero este consenso genérico suele basarse en definiciones ambiguas del concepto y en referencias todavía más indefinidas sobre su concreción. Se plantea su vinculación a cuestiones como la coordinación, la cooperación o la mejora de la comunicación entre las unidades de la organización. Aunque estos términos constituyen algunas de las manifestaciones de un funcionamiento transversal, deberían complementarse con referencias a la estructura orgánica y a su funcionamiento de acuerdo con los objetivos de la organización. A modo de concepto operativo a considerar, se puede entender la transversalidad en términos de opción organizativa orientada a introducir objetivos compartidos por el conjunto de la organización y que van más allá de los asignados a cada división u órgano sectorial (Serra 2004), pero que requieren de su participación efectiva para su consecución.

En otros términos, se puede entender la transversalidad como una nueva tendencia organizativa de carácter horizontal que busca la integración, la coordinación y la comunicación interdepartamental, caracterizada por proponer unos objetivos asumidos por todos los sectores de la organización 
pero que no son propios de ninguno de ellos sino generales del conjunto.

Así definida, la transversalidad se plantea como un modelo organizativo alternativo al tradicional para dar respuesta a los nuevos retos que se plantean a los gobiernos y a las administraciones públicas. Este modelo se define por contraponer el principio de coordinación horizontal al principio de jerarquía y coordinación vertical, y la creación de grupos de trabajo multidisciplinares a la especialización funcional pura. Pero aunque la transversalidad se conforme como una alternativa organizativa, se considera que no debe entenderse como una opción sustitutiva al modelo burocrático sino como una aproximación que lo complementa y lo enriquece.

En la citada contraposición cabe no obviar que en las administraciones públicas existen servicios transversales muy desarrollados, y un claro ejemplo lo constituyen las unidades de la tecnoestructura como encargadas de la gestión económica, los recursos humanos o las propias vinculadas a la implantación de las TIC. Pero dentro de la categoría de éstas ultimas conviene distinguir aquellas planteadas con un carácter operativo y de apoyo, con un enfoque altamente sectorializado y frecuentemente jerarquizado, de la existencia de unidades con un planteamiento de trabajo transversal que, para un ámbito estratégico como el desarrollo del e-gobierno, planteen estrategias basadas en los objetivos compartidos apuntados en la definición del concepto.

En dicha línea, cabe considerar que, más allá de plantearse como un instrumento de gestión o una solución técnica, la transversalidad implica el surgimiento de una nueva manera de entender las relaciones en la organización. Aunque complementaria a la existencia de determinadas dinámicas de funcionamiento, como se apuntó anteriormente, el impulso de la transversalidad supone incorporar nuevos valores y pautas de relación que inciden en el conjunto de unidades que conforman la administración pública.

En relación al enfoque de las transversalidad, las TIC representan una gran oportunidad para romper las lógicas sectoriales y avanzar en el desarrollo de dinámicas de diseño y gestión de carácter transversal. La creación de bases de datos únicas, intranets corporativas, servicios públicos electrónicos, extranets interadministrativas o páginas con contenidos creados de forma participativa son tan sólo algunos instrumentos operativos que, según se planteen, pueden fomentar la institucionalización de lógicas transversales, tanto a nivel interno como a nivel externo y relacional.

A nivel interno, las TIC pueden facilitar la distribución plural 
de la información y con ello contribuir a superar lógicas de gestión patrimonialista de la misma. A nivel externo y relacional, las tecnologías pueden contribuir a apoyar la actividad de prestación directa de servicios públicos y la coordinación de redes de actores que participan en las políticas y servicios públicos. En lógica de gobierno electrónico, las TIC pueden contribuir a fomentar valores democráticos y la participación ciudadana más enriquecida, generando espacios de relación y creación conjunta de respuestas desde el sector público. En definitiva, las TIC pueden constituir el sistema nervioso de la organización, un sistema dinámico y de geometría variable que puede potenciar muy activamente las lógicas organizativas de carácter transversal.

De todas maneras las TIC tienen una dimensión tecnológicay tecnocrática que requiere de liderazgo político y de contenidos. En este sentido, las TIC serán potentes en el fomento de la transversalidad cuando se planteen más allá de su concreción tecnológica y con una clara orientación estratégica integrada, en lógica de gobierno electrónico. Para ello resulta esencial pasar del nivel de discurso al nivel de la concreción, pero atendiendo tanto a los actores involucrados como a las instituciones vigentes y a la gestión del cambio necesaria para adecuarlas a los objetivos planteados.

\section{E-GOBIERNO Y TRANSVERSALIDAD: ORGANIZACIÓN, ROLES DIRECTIVOS Y ESTILOS DE RELACIÓN}

Para afrontar la complejidad inherente al estudio de la transversalidad en las administraciones públicas, resulta conveniente introducir la perspectiva neoinstitucionalista para poder caracterizar las diferentes dimensiones de cambio que incorpora. Desde este enfoque, se entiende por institución el conjunto de reglas, procesos y estructuras, pero también de rutinas, normas y valores (March y OlsenI 989, Kiser y Ostrom I982, Scott I 995) que vertebran las organizaciones y explican su funcionamiento. En este sentido, el concepto de institución enriquece y amplía el de organización, puesto que no solamente se ocupa de la dimensión formal, de las estructuras administrativas, de los objetivos y las reglas que gobiernan su funcionamiento interno y su relación con el entorno, sino que también incorpora de la dimensión cultural, informal, los comportamientos que se dan entre los miembros de la organización, así como la intersección entre estas dimensiones. En otros términos, las instituciones constituyen un conjunto interrelacionado de normas, valores, estructuras, rutinas y procesos, que se refuerzan mutuamente generando una suerte de "reglas del juego" que contribuyen a explicar tanto el comportamiento de los actores involucrados como el funcionamiento y los resultados efectivos del sistema (March y Olsen 1989, Kiser y Ostrom I 982 , Scott 1995). 
Partiendo de esta perspectiva, la transversalidad plantea un nuevo equilibrio dentro las administraciones, un cambio en los objetivos y las estructuras administrativas, pero también un cambio en las reglas del juego y en las pautas de comportamiento de los individuos y de las unidades. Y resulta especialmente complicado cambiar estas reglas del juego o instituciones, especialmente si tal como se ha apuntado anteriormente éstas cuentan con una importante tradición previa y el apoyo de distintos colectivos profesionales involucrados en su mantenimiento y reproducción (Lanzara I999).

Tal como se comentó en la introducción, las estructuras y dinámicas de funcionamiento de las administraciones públicas distan de favorecer dinámicas de funcionamiento transversal, a pesar de las exigencias del entorno social y económico y a pesar de la creciente presión de referentes externos en esta dirección (Mora, Morat y Ezpeleta 2006, Ramió y Salvador 2007).

La generación de proyectos transversales, habitualmente vinculados a un ámbito temático o territorial concreto, han sido una respuesta habitual con importantes problemas de consolidación e institucionalización más allá de la experiencia puntual (Ramió 2005). La incorporación de servicios públicos electrónicos vinculados a un aplicativo sectorial podría ser un ejemplo de este tipo de enfoque.

Sin embargo, el cambio que representa introducir la lógica transversal en ámbitos que inciden en el conjunto de la organización, y que contribuya por tanto al cambio institucional asociado, requiere de estratégicas más integradas. Siguiendo el ejemplo del gobierno electrónico, dichas estrategias deberían abordar de forma simultánea: I) la organización, los objetivos y las estructuras administrativas, y 2) la gestión de la transversalidad, en términos de roles directivos, y estilos de relación.

\section{LA ORGANIZACIÓN DE LA TRANSVERSALIDAD: EL PAPEL DE LAS UNIDADES TRANSVERSALES Y LA GESTIÓN EN RED}

La transversalidad en tanto que herramienta organizativa incorpora una nueva definición de la relación entre objetivos y recursos que, idealmente, se asocia a una transformación integral de las dinámicas internas de la organización para afrontar el desarrollo de respuestas integrales y atender a nuevas demandas complejas.

Pero ante las dificultades para lograr transformaciones de tal alcance, a menudo se opta por crear unidades orgánicas con una orientación de carácter transversal. Estos órganos suelen ser los impulsores de políticas 
transversales para el conjunto de la organización, y presentan unas características que los diferencian del resto de la estructura administrativa.

Cabe no confundir estas unidades, planteadas en términos de impulsoras de estrategias integradas de gobierno electrónico, con las unidades de apoyo informático que, con un enfoque más operativo, suelen dedicarse a la vertiente más tecnológica de la implantación de sistemas de información y comunicación.

Por el contrario, las unidades transversales a las que se refiere el epígrafe, no son unidades de gestión operativa ni de producción de servicios, sino que se sitúan en un nivel estratégico y colaboran con las unidades funcionales (que se acostumbra a mantener sectorializadas). Sus funciones son de diseño, planificación y evaluación de las políticas vinculadas al gobierno electrónico, así como la colaboración en la implantación de sus concreciones a cada ámbito sectorial. Las unidades transversales actúan como unidad de diseño y a la vez de apoyo de las unidades sectoriales, como una consultora interna especializada en un ámbito de políticas que tiene un impacto transversal en las diferentes áreas de la organización. La aportación de las unidades transversales se basa en su conocimiento experto, en la información, pero también en el diseño de instrumentos de gestión adaptados y adaptables a las diferentes necesidades sectoriales. Siguiendo este argumento, las funciones más habituales de las unidades transversales se pueden agrupar en:

I. Nodo de información: captura, produce y difunde la información al resto de la organización. Crea conocimiento y expertise para el ámbito transversal concreto.

2. Nodo de coordinación estratégico: no solamente identifica los objetivos de las actuaciones transversales, tanto a nivel corporativo como a nivel sectorial, sino que también diseña políticas, desarrolla metodologías de trabajo y estructura las actuaciones.

3. Nodo de red intraorganizativa que implementa la estrategia transversal: la unidad gestiona las redes relacionales internas y externas, y para hacerlo desarrolla canales de comunicación, instrumentos de coordinación ad-hoc, tanto formales como informales, sistemas de evaluación de resultados y de rendimiento de cuentas.

De acuerdo con esto, las unidades transversales tienden a tener unas dimensiones reducidas, con un personal especializado en aquellas actividades que aportan valor a la implementación de la estrategia: el diseño, la planificación, el seguimiento y la evaluación de la actuación 
pública.

En segundo lugar, las unidades transversales acostumbran a ocupar una posición diferenciada dentro del organigrama. Por norma general, dependen directamente del nivel de decisión político, sin integrarse dentro de la cadena de mando. Esta adscripción orgánica se asocia tanto a su papel estratégico en la implementación de las políticas transversales como a la necesidad de legitimarse ante las unidades sectoriales que llevarán a cabo la implementación de las líneas de actividad. Aunque esta adscripción puede llevar a considerarlas como unidades staff o de apoyo, debe considerarse que también presentan dos diferencias importantes: por un lado no prestan apoyo al inmediato jerárquico tal y como hacen las unidades staff tradicionales, sino a las unidades sectoriales; por otro lado, las unidades transversales no se limitan a ejercer un rol de apoyo, sino que participan en el proceso operativo, se coordinan con los ámbitos sectoriales para implementar las políticas transversales de gobierno electrónico.

En tercer lugar, la relación de la unidad transversal con las unidades sectoriales que gestionan en el nivel funcional y operativo el despliegue de la estrategia de gobierno electrónico no se basa en mecanismos de coordinación jerárquica, sino en mecanismos de coordinación horizontal, de cooperación y adaptación mutua. Es en este sentido que las unidades transversales representan claramente la idea de organización en red: ejercen de nodo de la red de implementación de las políticas transversales, liderándolas pero sin llegar a dirigir las unidades sectoriales sino coordinándose con ellas. Se podría afirmar que las unidades transversales disfrutan de cierta autoritas -basada en su conocimiento experto en el ámbito de gestión transversal concreto, pero también por la legitimación que los confiere su posición dentro el organigrama- pero no tienen la potestas sobre las unidades sectoriales que implementan la política pública transversal, de forma que la eficacia y la eficiencia de las actuaciones impulsadas se vincula con la capacidad de coordinación de las unidades transversales.

El funcionamiento en red de las unidades transversales en su relación con las unidades sectoriales plantea una serie de elementos críticos a la hora de considerar la transversalidad como una solución organizativa más eficaz y eficiente que la estructura tradicional jerárquica (Kickert, Klein y Koppenjan 1997).

I. El coste de la coordinación aumenta con el funcionamiento en red de las unidades transversales. A diferencia de las estructuras verticales, en las cuales los flujos de información siguen principalmente una sola dirección, de arriba abajo, en las estructuras en red se generan dinámicas de retroalimentación que aseguran una mayor sensibilidad 
a las necesidades reales, pero que implican unos costes mayores. Por otro lado, en las estructuras verticales las decisiones atraviesan -supuestamente- más rápidamente la estructura administrativa, mientras que en estructuras de red es necesario establecer transacciones, negociar y llegar a acuerdos, por lo que el proceso de decisión y de implementación se vuelve más lento y laborioso.

2. El establecimiento de responsabilidades es otro punto crítico en la comparación entre el modelo de coordinación jerárquico y el modelo de coordinación horizontal en red. Mientras que el establecimiento de cadenas de mando permite identificar las líneas de responsabilidad en las estructuras tradicionales, el rendimiento de cuentas en las estructuras en red parece más difuso, distribuido de forma menos específica en el conjunto de la red.

3. Vinculado al punto anterior, en la valoración de los resultados logrados por las estructuras que trabajan en red hace falta también tener en cuenta las dificultades para identificar los elementos que los explican. En contraposición, las estructuras fragmentadas y sectorializadas tradicionales permiten atribuir con mayor claridad la aportación de cada una de las unidades en la cadena de valor.

De todas maneras, las tres críticas pueden redefinirse a partir del potencial que aporta la gestión en red con criterios transversales. Por ejemplo, los costes de coordinación pueden ser contrapuestos a las ganancias obtenidas a partir del surgimiento de respuestas integradas más adecuadas a las cuestiones a tratar. Y las redes pueden reforzar el rendimiento de cuentas de la organización a través de la inclusión de la ciudadanía como un agente más, o bien a través de la mayor transparencia que se da entre los integrantes de la red. Así, los elementos críticos presentados lo son en tanto que líneas de reflexión, y prefiguran la importancia de la gestión transversal para asegurar unas organizaciones más eficaces y eficientes.

\section{LA GESTIÓN DE LA TRANSVERSALIDAD: ROLES DIRECTIVOS Y MODELOS DE RELACIÓN}

Una segunda dimensión de impulso a la transversalidad va más allá del funcionamiento de las actuaciones impulsadas por las unidades transversales para centrarse en la relación que estas establecen con las unidades sectoriales. En este punto los mecanismos de relación y de influencia con el resto de órganos sectoriales juegan un papel preponderante en la explicación de los resultados de la transversalidad.

La función directiva es un primer elemento clave para entender el 
cambio que se plantea, y se hace necesario definir un perfil directivo transversal diferenciado del perfil de directivo sectorial más extendido. El directivo transversal se caracteriza por combinar la dirección de su propio equipo de trabajo pero a la vez coordinar la red intraorganizativa asociada al desarrollo de las políticas transversales. Entre otros, el perfil de directivo transversal se caracteriza por: I) hacer un menor uso de los instrumentos de gestión operativa y un uso más intensivo de los elementos de análisis y diseño estratégico, de la gestión relacional y de la evaluación, y 2) desarrollar intensivamente los instrumentos de relación y coordinación con los directivos sectoriales con los que no tiene vinculación jerárquica.

Esta segunda dimensión resulta clave en la explicación de los resultados de las políticas transversales: los directivos transversales no aspiran a fortalecer su área de mando, a crecer en recursos y dotaciones, sino a fortalecer la relación con el resto de áreas, a ejercer el liderazgo en las políticas transversales a través de su conocimiento experto, pero también a través de la capacidad de persuasión. En otros términos, redefinen el concepto de poder en la organización y a menudo por ello suelen ser más cuestionados desde las posiciones más consolidadas en el modelo burocrático.

En un segundo nivel vinculado a competencias en gestión de la transversalidad, las actividades asignadas a las unidades transversales se vinculan con una serie de metodologías de trabajo:

I. La gestión transversal requiere de un alto nivel disponibilidad y de transparencia de la información, no solamente en el proceso decisional, sino también en la relación con las unidades sectoriales. Por un lado, la legitimación de las unidades transversales se basa en su conocimiento experto, pero por otro lado, la transparencia y la fluidez en la comunicación constituyen elementos de primer orden para facilitar la coordinación y evitar desconfianzas.

2. La relación entre las unidades transversales y las sectoriales se da tanto en el nivel formal como en el informal. La gestión transversal permite aflorar el elemento informal de la organización, los liderazgos no basados en la posición jerárquica sino en la posesión de determinadas habilidades, recursos y conocimientos, y el logro de soluciones de forma conjunta. Además, el grado de formalización orgánica de la relación no viene prefijado sino que es susceptible de tomar diferentes formas.

3. La gestión transversal supone un esfuerzo para visualizar la cooperación en términos sinérgicos, una ganancia tanto para las unidades sectoriales como para las transversales. De acuerdo con este supuesto de partida, se prioriza el logro de soluciones adaptadas a las necesidades de las 
partes, con grados de implicación variable, tanto en relación a los objetivos de la política transversal como en relación a la implicación de las diferentes unidades sectoriales.

4. En cuanto a la transparencia y el fomento de la implicación, la evaluación de los resultados de las políticas transversales constituye un proceso participativo que involucra tanto la unidad transversal como a las sectoriales. De este modo, se visualizan y se comparten los resultados de la transversalidad, a la vez que se facilita el rendimiento de cuentas dentro la red. En ningún caso, la unidad transversal se debería erigir en auditora de los resultados de las unidades sectoriales.

En consonancia con los aspectos señalados, la utilización estratégica de los diferentes instrumentos vinculados a las TIC, pero en clave de gobierno electrónico, es un elemento crucial para un verdadero funcionamiento en red, no tan sólo porque facilitan la transparencia y permiten el establecimiento de relaciones de confianza entre las partes, sino también porque aseguran un funcionamiento integrado de la red, una mayor adaptabilidad a las contingencias, pero también el establecimiento de líneas de responsabilidad y control mutuo.

La gestión transversal es intensiva en el uso de canales de información y comunicación y en este sentido cabe destacar que la propia implantación de estrategias integradas de gobierno electrónico propicia el cambio de enfoque y sus propios contenidos se convierten en elementos facilitadores de la gestión transversal. Por un lado, los cambios de las estructuras administrativas que impulsan las TIC no solamente se centran en la mejora de los procesos de trabajo, sino también en una mayor difusión de la información dentro la organización, lo que puede contribuir a incrementar la transparencia en la gestión. Por otro lado la introducción de las TIC facilita el establecimiento de relaciones informales de enlace más flexibles, no basadas exclusivamente en la posición en el organigrama de los individuos, y que incrementan en el potencial de coordinación en su actuación sectorial.

Pero la gestión de la transversalidad en su concreción en la estrategia de gobierno electrónico no se limita a la dimensión interna de la organización, sino que el funcionamiento en red propio de las unidades transversales también comporta la relación con el entorno a la organización en un doble sentido. Por un lado, las unidades transversales deben ser capaces de captar demandas externas e internas, generar nueva información y transferir conocimiento. Por otro lado, las unidades transversales pueden impulsar la relación con otras administraciones y con las entidades privadas a las que se externaliza la prestación de los servicios públicos y de este modo velar 
por la eficacia en la ejecución de las iniciativas transversales vinculadas a la creación de valor público.

\section{LA CULTURA DE LA TRANSVERSALIDAD}

Tal y como se apuntaba en el apartado anterior al vincular el modelo de administración relacional con las estrategias e instrumentos por fomentar la transversalidad, ésta no debe considerarse sólo como una mera herramienta de gestión sino también como una nueva manera de definir las dinámicas de trabajo y de entender las relaciones intra e interorganizativas. Así, la transversalidad no solamente comporta un cambio en las estructuras administrativas, en sus funciones, en los procesos de trabajo, los instrumentos de gestión o en los perfiles directivos, sino que implica un cambio en los valores de la organización y en las pautas de relación entre los individuos y las unidades. El funcionamiento en red propio de las unidades transversales permite apuntar hacia una cultura organizativa de la transversalidad, que se fundamenta en unos principios, valores y normas sustancialmente diferentes de la cultura organizativa burocrática tradicional.

De hecho, la cultura de la transversalidad supone una reacción a las limitaciones de las estructuras burocráticas y a su énfasis en los mecanismos de coordinación jerárquica, que generan una serie de disfunciones (Echevarria 200I).

I. El establecimiento de líneas de mando verticales muy extensas acaba comportando una pérdida de responsabilidad en cada uno de los niveles de la línea, principalmente en los intermedios. Cada nivel de responsabilidad depende de un de superior y un de inferior de forma que la atribución de responsabilidades acaba siendo difusa.

2. La dificultad en el establecimiento de responsabilidades se vincula con un problema más estructural, los déficits de funcionamiento de los canales de comunicación de las estructuras jerárquicas, que deriva a su vez en la dificultad para establecer de forma clara los resultados de cada nivel y unidad. Además, este déficit de información hace que las unidades se perciban en términos de competencia y de desconfianza mutua.

3. Las estructuras jerárquicas desincentivan el surgimiento de la creatividad de los miembros de la organización en todos los niveles, así como la retroalimentación de la información y del conocimiento que se genera. La restricción con la que se definen los puestos de trabajo supone un freno para el desarrollo del potencial de los diferentes miembros, que 
de este modo tienden a desmotivarse. Por otro lado, el conocimiento que se genera en la base operativa, fruto del contacto con las demandas de los usuarios, se pierde dentro de la organización.

Así, las estructuras burocráticas favorecen un tipo de cultura organizativa en la que se debilita el rendimiento de cuentas y la responsabilidad, el funcionamiento por resultados no está claro, y donde se desaprovecha tanto el potencial de los recursos humanos de la organización como la información que se produce internamente.

Frente de este diagnostico, la transversalidad y su opción por la coordinación horizontal implican unos cambios que suponen la aparición de una nueva cultura organizativa, la cultura de la transversalidad, que poniendo en valor la dimensión informal de la organización, apuesta especialmente por valores como la confianza (Brugué 2003). Desde la dimensión informal la cultura de la transversalidad pretende movilizar recursos, canales de información y un capital humano que se pueden integrar a la organización mediante las estructuras transversales. Además, al incorporar los mecanismos de coordinación propios de la dimensión informal se reduce el coste a la vez que se otorga mayor flexibilidad al funcionamiento de las organizaciones: los sistemas de relación informales, basados en canales de información lateral, negociaciones y normas de reciprocidad que se adaptan mejor a las necesidades cambiantes del funcionamiento operativo en entornos complejos.

Siguiendo las líneas críticas de la cultura organizativa burocrática, la cultura de la transversalidad presentaría las siguientes características:

I. La cultura organizativa transversal enfatiza el sentido compartido de la organización, una visión de conjunto de sus objetivos, que a la vez se vincula a una ética revitalizada de la responsabilidad. Los mecanismos de comunicación interna que pueden asociarse a un desarrollo integrado y estrategico de gobierno electrónico mediante intranets corporativas pueden contribuir a reforzar este punto a nivel operativo.

2. La cultura de la transversalidad se expresa a través de sistemas de coordinación horizontal, que favorecen la participación de los diferentes miembros de la organización. En este sentido, la transversalidad se configura como una cultura participativa que motiva los individuos y los ofrece opciones para desarrollar. Siguiendo el enfoque estratégico del gobierno electrónico, la utilización de espacios de trabajo colaborativo, con lógicas de web 2.0 dentro de las intranets corporativas puede favorecer la operativización de esta tendencia. 
3. Una cultura de la interdependencia de la organización. La cultura organizativa se ha de entender como la vinculación de los miembros de la organización. En el caso de la transversalidad esta vinculación tiende a asociarse a un sentido más democrático, igualitario y solidario de concebir la organización. El impulso de modelos de gestión por procesos como referente de apoyo al despliegue del gobierno electrónico en la dimensión interna puede contribuir en esta línea, articulando las líneas de actividad no en función de la adscripción orgánica sino a partir de los flujos de trabajo identificados en la organización.

4. La cultura de la transversalidad se fundamenta en el conocimiento y la información, no en la dominación y la distribución selectiva de información: la cooperación se fundamenta en el intercambio de información especializada, pero a la vez la autoridad tiende a basarse más en el conocimiento experto y en la capacidad de movilización que en la mera posición jerárquica. Cada vez más la lógica de redes sociales, y sus concreciones en las comunidades virtuales de profesionales, también inciden en la misma orientación, por lo que deberían considerarse en una estrategia de gobierno electrónico.

Esta primera aproximación a la cultura de la transversalidad debe enmarcarse en los cambios en la organización y en la gestión apuntados anteriormente, y que tan sólo son el punto de partida de un proceso de ajuste de la realidad de nuestras administraciones.

\section{UNA REFLEXIÓN FINAL A MODO DE CONCLUSIÓN}

Uno de los importantes riesgos asociados al desarrollo de las iniciativas relacionadas al gobierno electrónico es reproducir pautas de funcionamiento de las organizaciones públicas introduciendo intensivamente la tecnología. Introducir aplicativos tecnológicos que refuercen automatismos en una administración públicaquefunciona siguiendo rígidas compartimentaciones que impiden afrontar problemas de política pública con la necesaria visión integral, difícilmente van a generar cambios sustantivos en sus capacidades de actuación. La introducción de tecnología suele contener cierto halo de modernidad y representa una magnífica ocasión para replantear las dinámicas de funcionamiento de las organizaciones, pero para ello se requiere de una visión previa sobre hacia donde debe cambiarse $y$, la propuesta por la transversalidad puede ser una idea-fuerza que permita optimizar la introducción de cambios.

Pero el reto al que se enfrentan las administraciones públicas actuales también es el de convertir el ambicioso discurso de la transversalidad en una realidad aplicable a todos los ámbitos de gestión pública, de forma que 
permita mejorar la capacidad de respuesta de los organismos públicos ante la creciente complejidad de las necesidades sociales y de los intereses de la ciudadanía.

El impulso y la implantación de iniciativas de gobierno electrónico en las administraciones públicas requieren de estrategias basadas en el modelo descrito de la transversalidad si realmente se pretende que constituyan un verdadero cambio institucional. En demasiadas ocasiones la implantación de las TIC se ha limitado a la introducción de meros aplicativos que, partiendo de enfoques excesivamente tecnológicos, han dejado de lado la transformación organizativa e institucional que potencialmente podían ofrecer. La revalorización del enfoque de la transversalidad ofrece, en estos términos, una oportunidad para replantear estrategias a partir de un nuevo estilo de trabajo y relación entre las organizaciones públicas y los profesionales que las integran. Para ello resulta esencial replantear las estrategias de gobierno electrónico considerando tanto su valor operativo como lo que suponen de oportunidad para asentar nuevas instituciones en las administraciones públicas. 


\section{REFERENCIAS}

Bellamy, Ch. y Taylor, J. A. (1998). Governing in the Information Age. Buckingham: Open University Press.

Bekkers, V. J. J. M. y Korteland, E. (2006). Governance, ICT and the Innovation Agenda of Public Administration: a Comparison of Some European Policy Initiatives. En Bekkers, V. J. J. M., van Duivenboden, H. y Thaens, M. (Eds), Information and Communication Technology and Public Innovation. Amsterdam: IOS Press.

Brugué, Q. (2003). Ėtica i Valors per Millorar i Transformar l'Administració Pública. Llibre Blanc de la Funció Pública Catalana. Barcelona: Generalitat de Catalunya.

Comisión Europea. (2006). Interoperabilidad de los Servicios Paneuropeos de Administración Electrónica. Bruselas: Comisión Europea.

Criado, I. y Ramilo, M. C. (200I). Definiendo la E-Administración: Las Páginas Web de las Administraciones Públicas. Gestión y Análisis de Póliticas Públicas, 22, I 23-138.

Criado, I., Ramilo, M. C. \& Salvador, M. (2002). La Necesidad de Teoria(s) sobre Gobierno Electrónico. Una Propuesta Integradora. Ensayo ganador de la mención honorífica del "XVI Concurso de Ensayos y Monografías sobre Reforma del Estado y Modernización de la Administración Pública” sobre Gobierno Electrónico organizado por el CLAD.

Dunleavy, P. y Margetts, H. (2007). Government on the Internet. Progress in Delivering Information and Services Online. Londres: National Audit Office.

Echebarría, K. (200I). Capital Social, Cultura Organizativa y Transversalidad en la Gestión Pública. Ponencia presentada en el VI Congreso Internacional del CLAD sobre la Reforma del Estado y la Administración Pública, noviembre, Buenos Aires.

Kiser, L. y Ostrom, L. (1982). The Three Worlds of Action: A Metatheoretical Synthesis of Institutional Approaches. En Ostrom, E. (Ed.), Strategies of Political Inquiry. Beverly Hils: Sage.

Kickert, W. J. M., Klijn, E. H. y Koppenjan, J .F. M. (Eds.). (I997). Managing Complex Networks. Strategies for the Public Sector. Londres: Sage.

Lanzara, G. F. (1999). Por Qué es tan Difícil Construir las Instituciones. 
Desarrollo Económico, 38 (I 52), 925-952.

March, J. G. y Olsen, J. P. (1989). Rediscovering Institutions. Nueva York: Free Press.

Mesa del Olmo, A. (2007). La Administración Electrónica en el Ámbito Local del País Vasco: El Desarrollo de las Páginas Web Municipales. Revista Vasca de Administración Pública, 77, I 33-176.

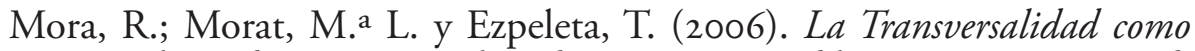
Impulsora de Mejoras en la Administración Pública en un Contexto de Desarrollo de un Sistema de Servicios Locales. Barcelona: Ediciones del Serbal.

Peters, B. G. (1999). Institutional Theory in Political Science: The "New Institutionalism". Londres: Painter.

Ramió, C. (2005). Las Debilidades y los Retos de la Gestión Pública en Red con una Orientación Tecnocrática y Empresarial. Sistema. Revista de Ciencias Sociales, I 84-I8 5, 87-106.

Ramió, C. y Salvador, M. (2007). El Disseny Institucional de l'àmbit de la Participació Ciutadana a les Corporacions Locals: Definició Conceptual i Propostes d'actuació. Diputació de Barcelona. Barcelona.

Ramió, C., Salvador, M. y García, O. (2007). Los Determinantes y la Gestión de la Externalización en Cataluña. Barcelona: Fundació Pi i Sunyer.

Salvador, M., Cortés, R. y Sánchez, R. (2004). Els Ajuntaments de Catalunya a Internet: Un Estudi Comparat de les Pagines Web (20002003). Barcelona: Universitat Pompeu Fabra.

Salvador, M. (2005a). Integrando el Gobierno Electrónico en el Ámbito Local: El Alcance de la Transformación más allá de las Modas. En Sancho, D. (Coord.), Sociedad de la Información y Gobierno. Málaga: Instituto Andaluz de Administración Pública.

Salvador, M. (2005b). Reflexiones sobre la Experiencia Catalana de Gobierno Electrónico en el Ámbito Local: Una Visión desde las Instituciones. Ponencia presentada en De las Administraciones en la Red a los Gobiernos y Sociedad en Red: Jornadas de estudio sobre el Gobierno electrónico, mayo, Leioia. . (2007). La Dimensión Interna del Gobierno Electrónico y el Impacto de la E-Administración en la Gestión de los Recursos Humanos: ¿Están Cambiando las Reglas del Juego? Administración y Ciudadanía, 2 (3), 
SALVAdor

I $85-198$.

Serra, A. (2004). La Transversalitat en la Gestió de Polítiques Públiques. Barcelona: Diputació de Barcelona, Síntesi 7.

Scott, W. R. (I995). Institutions and Organizations. Thousand Oaks: Sage.

Recibido: 06-09-2011

Aceptación de la versión final: 05-03-2012 